\title{
EchoGéo
}

23 | 2013

Entre l'ancien et le nouveau monde. La transcendance du lieu et de l'espace en géographie vinicole contemporaine

\section{Parcours de géographe : Antoine Haumont, cinquante ans de pratiques de l'interdisciplinarité et de l'intercognitivité}

\section{Alexis Sierra}

\section{(2) OpenEdition}

Journals

Édition électronique

URL : https://journals.openedition.org/echogeo/13324

DOI : 10.4000/echogeo. 13324

ISSN : 1963-1197

Éditeur

Pôle de recherche pour l'organisation et la diffusion de l'information géographique (CNRS UMR 8586)

\section{Référence électronique}

Alexis Sierra, «Parcours de géographe : Antoine Haumont, cinquante ans de pratiques de

l'interdisciplinarité et de l'intercognitivité », EchoGéo [En ligne], 23 | 2013, mis en ligne le 05 juin 2013,

consulté le 11 août 2021. URL : http://journals.openedition.org/echogeo/13324 ; DOI : https://doi.org/

10.4000/echogeo.13324

Ce document a été généré automatiquement le 11 août 2021.

EchoGéo est mis à disposition selon les termes de la licence Creative Commons Attribution - Pas d'Utilisation Commerciale - Pas de Modification 4.0 International (CC BY-NC-ND) 


\title{
Parcours de géographe : Antoine Haumont, cinquante ans de pratiques de l'interdisciplinarité et de l'intercognitivité
}

\author{
Alexis Sierra
}

1 Le rôle du géographe dans la société et sa place dans le champ des savoirs est sans cesse interrogé par la discipline. Dans une période où l'incertitude gagne le milieu académique des sciences humaines et sociales, entre finalité professionnelle et finalité scientifique, comme si l'une et l'autre devaient être antinomiques, l'examen du parcours de Antoine Haumont montre à travers plus de cinquante ans d'enseignement et de recherche en géographie, que l'analyse géographique a été non seulement reconnue par d'autres disciplines mais aussi par des décideurs et certains milieux professionnels. Il montre également que les évolutions politiques (suite du mouvement de mai 68 , alternance de 1981) et celles de l'action publique (dans le domaine de l'aménagement $\mathrm{du}$ territoire et de l'enseignement) surdéterminent le rôle du géographe. Ce parcours montre enfin une génération de chercheurs et d'enseignants qui se constitue progressivement en réseau épistémique à l'occasion du développement des sciences sociales porté par les acteurs publics, des ministères aux institutions académiques.

2 Le texte qui suit a été élaboré à partir d'une série d'entretiens et d'échanges avec Antoine Haumont qui a également fourni un ensemble de documents de référence. Cet exercice d'écriture assume pleinement la subjectivité du point de vue, puisque l'objectif est de montrer comment un géographe a pu ressentir son positionnement parmi le champ global des savoirs, que ceux-ci aient procédé du milieu scientifique ou du milieu des opérateurs.

Entre le début de ses études de géographie en 1954 et sa retraite de l'Université de Paris 7- Denis Diderot en 2000, Antoine Haumont passe d'une formation classique à une confrontation répétée avec de multiples disciplines (sociologie, démographie, 
architecture, sciences de l'ingénieur) et avec les besoins de l'opérationnel. Son savoir de géographe se trouve ainsi en interrelation permanente avec d'autres savoirs scientifiques dans une perspective interdisciplinaire et avec le savoir des responsables politiques et administratifs dans une perspective intercognitive non nécessairement programmée et formalisée mais se faisant au gré des études et des enseignements.

\section{Une formation dans un contexte universitaire parisien en ébullition}

4 Se destinant à des études d'histoire alors qu'il était en hypokhâgne à Louis Le Grand, l'orientation d'Antoine Haumont vers la géographie puise sa source dans les enseignements dispensés pour des modules d'équivalence, notamment par de jeunes assistants qui lui ouvrent de nouveaux horizons, Marcel Roncayolo et Yves Lacoste. Pour un jeune étudiant engagé dans la cité, l'Institut de géographie, rue Saint-Jacques apparaît également comme un lieu plus convivial et plus ouvert que ne l'est la Sorbonne proprement dite où évoluent les historiens. La rue Saint-Jacques est un lieu de rencontres entre une génération de professeurs et une génération d'étudiants marqués par la guerre d'Algérie pour lesquels l'engagement à gauche passe souvent par l'adhésion au Parti Communiste Français. Antoine Haumont, qui milite activement à l'UNEF, y découvre la géographie des régions coloniales et les problématiques tiersmondistes. C'est lors d'une des réunions au «115», le siège de l'association des étudiants d'Afrique du Nord est situé au 115 du boulevard Saint-Michel, qu'il entend pour la première fois Henri Lefebvre, figure universitaire de la gauche parisienne, également communiste. A l'Institut de géographie, il côtoie Michel Coquery et Armand Frémont qui vont marquer de manière décisive certaines étapes de son parcours professionnel. Jean Dresch fait partie de ses professeurs.

5 Cependant pour lui, le géographe marquant de cette époque reste Pierre George dont il suit, avec admiration et enthousiasme, les cours. Celui-ci est alors un géographe déjà largement reconnu et Antoine Haumont en garde le souvenir d'un professeur qui remplissait régulièrement l'amphithéâtre de l'Institut de géographie, dressant une grande fresque des sociétés urbaines dans le monde, de manière vivante et concrète par sa connaissance directe des pays d'Europe centrale et orientale, de l'URSS, des EtatsUnis. C'est pourquoi intéressé par l'étude d'une convergence de flux déterminés par un pôle industriel il le sollicite pour le diriger en maîtrise, en lui proposant une «Étude des migrations de population à Boulogne-Billancourt » (1957). Il obtient ensuite le CAPES puis l'agrégation de géographie, et comme beaucoup de ses condisciples commence sa carrière dans le secondaire, au lycée de Louviers (1959-1962) puis en classe préparatoire au lycée Paul Valéry à Paris. Rapidement, les besoins d'enseignement dans une université qui voit croître fortement ses effectifs d'étudiants conduisent Pierre George à le solliciter pour devenir assistant en 1965.

\section{Une recherche interdisciplinaire avec Henri Lefebvre.}

6 Entre-temps, sa fréquentation des sociologues, notamment grâce à son épouse Nicole, le conduit en 1962 à participer à la fondation de l'Institut de Sociologie Urbaine (ISU) dirigé par Henri Lefebvre. Un grand nombre études urbaines sont, à l'époque, aussi bien menées au sein d'institutions scientifiques que d'associations loi 1901 ayant un objectif 
de recherche : Paul-Henri Chombart de Lauwe, sociologue au CNRS, a ainsi créé en 1959, le Centre d'Ethnologie Sociale. Au CNRS, le Centre de Sociologie Urbaine, d'orientation clairement marxiste, réunit des sociologues comme Jean Lojkine, Christian Topalov, Manuel Castells. Dans ce panorama, l'ISU était une association (loi 1901) dont la création a reposé sur plusieurs facteurs. Une première dynamique vient du développement des recherches publiques sur les villes et les sociétés urbaines dans la France des années 1960. Diverses compétences sont alors associées, celles proprement scientifiques des Universités et du CNRS et celles des administrations publiques en charge des secteurs de la construction et de l'aménagement. En même temps, la diversité des thèmes de recherche s'affirme. Parmi ces thèmes figurent les analyses du philosophe Henri Lefebvre sur la vie quotidienne, incitant à connaître et à comprendre les habitants, leurs pratiques et leurs modes de vie. Il a notamment publié en 1961 "Critique de la vie quotidienne». Enfin, les recherches sur la ville offrent des opportunités de travail et de carrière à de jeunes chercheurs. C'est notamment le cas de la petite équipe animée par les sociologues Henri Raymond et Nicole Haumont, équipe qui crée l'ISU et demande à Henri Lefebvre de le présider. Outre divers sociologues, le groupe comprend des ingénieurs provenant de l'Institut d'Aménagement et d'Urbanisme de la Région Parisienne (qui devint ultérieurement l'IAU). Antoine Haumont est le géographe de l'équipe.

7 Une des premières recherches de l'équipe porte sur l'habitat individuel en France. Elle est commandée par le Centre de Recherche d'Urbanisme qui vient d'être créé en 1962 en co-tutelle par le Ministère de la Construction et le Ministère de l'Education nationale. En son sein, un groupe de travail dirigé par le géographe Pierre George veut comparer les grands ensembles de logement et les maisons individuelles. La recherche de l'ISU est publiée en 1966, sous le titre "L'habitat pavillonnaire » avec une préface de Henri Lefebvre. La recherche développe une analyse des dimensions à la fois historiques, géographiques et culturelles des maisons individuelles. La contribution d'Antoine Haumont a surtout porté sur les sites et les types de cet habitat. L'ouvrage, primé médaille de bronze du CNRS, prit place dans le débat sur les avantages et les inconvénients des maisons individuelles. Il bousculait les idées reçues de l'époque qui voulaient que ce mode d'habiter était contraire à la modernité, favorisait l'individualisme et l'étalement urbain.

De 1966 à 1986, l'ISU a continué des recherches sur les pratiques urbaines et Antoine Haumont a pu travailler sur l'habitat, sur les espaces du travail, sur les équipements culturels et sur la mobilité quotidienne. Ses publications montrent clairement une géographie dans laquelle les pratiques quotidiennes dans les villes, en particulier dans l'agglomération parisienne forment un objet central.

\section{La construction de la suprafonctionnalité des sciences de l'ingénieur}

Dans les années soixante, la vision techniciste de l'équipement et de l'aménagement est remise en cause. Les études menées au sein du Ministère de l'Équipement montrent que les solutions des ingénieurs n'aboutissent pas toujours aux résultats escomptés, en particulier lorsque les habitants et les usagers n'adoptent pas les innovations techniques. 
10 Les membres de l'École Nationale des Ponts et Chaussées (ENPC) estiment alors qu'à côté des formations aux sciences de l'ingénieur, il faut développer les sciences sociales. L'ENPC dispensait déjà des cours d'économie et de droit mais essentiellement en relation avec la production de techniques (normes, coûts). En revanche, il n'y avait pas de cours en sciences sociales qui auraient permis de réfléchir à l'adaptation des techniques aux conditions et aux modes de vie. L'idée est donc d'introduire dans la formation des ingénieurs des connaissances supra-fonctionnelles c'est-à-dire qui obligent à penser les réseaux d'infrastructure en relation avec la société qui les utilise. En 1965, l'École choisit ainsi de créer des enseignements d'aménagement qui donnèrent une place aux géographes. D'abord à Michel Coquery, puis à Antoine Haumont comme maitre de conférences (1973-1982) puis comme professeur (1983-2000). C'est l'occasion de faire découvrir aux futurs ingénieurs les transformations et les tensions de l'espace français de l'époque. Fidèle à la tradition de confrontation au terrain, il organise des voyages d'étude pour analyser les modes de vie et les conditions d'urbanisation de différentes villes françaises et européennes, en particulier à Barcelone, Berlin et Budapest. Le contexte politique de l'époque était propice à des réflexions et à des comparaisons originales, Berlin étant alors coupée en deux et Budapest marquée par le modèle de développement communiste. Concernant la métropole allemande, Antoine Haumont rappelle que des ingénieurs avaient pu trouver l'ordre de Berlin-Est préférable aux agitations alternatives de Berlin-Ouest...

\section{L'organisation de l'interdisciplinarité avec les architectes}

En 1982, le nouveau gouvernement de gauche commande un rapport sur les sciences de l'homme et de la société à l'anthropologue Maurice Godelier. Il y préconise notamment la création de sections pluridisciplinaires ou de « fronts intersciences» (Godelier, 1982). Le démographe (et mathématicien) Hervé Le Bras, qui le conseille, propose de créer une section pour l'architecture et l'urbanistique. Dans le rapport, ce dernier terme est une des échelles de l'architecture et renvoie à l'étude de "l'espace des villes, leur morphologie, les typologies, l'esthétique » et fait écho à l'anglais « urban design » alors qu'urbanisme se traduirait par «urban planning» et selon l'auteur "privilégie l'analyse sociale, politique et économique des villes». Plus globalement, le rapport indique qu'au «CNRS, on ressent l'absence de chercheurs capables de penser l'espace et ses transformations » soulignant un éclatement du discours sur l'espace en fonction des disciplines. La proposition est retenue et sa mise en œuvre est confiée à Antoine Frémont, directeur adjoint du département des sciences sociales, qui demande à Antoine Haumont d'étudier le dossier. Ce dernier anime un groupe de travail constitué de personnes provenant de trois domaines: des chercheurs en sciences physiques pour l'ingénieur parmi lesquels des acousticiens et des spécialistes de la résistance des matériaux ainsi que des informaticiens ; des chercheurs provenant du département de sciences humaines et sociales ; enfin, des enseignants en architecture qui étaient déjà familiarisés avec les méthodes de recherche et qui ont dû être sollicités auprès de différentes écoles d'architecture (de Rennes, de Marseille, etc.). L'absence de thèses d'architecture validées par un diplôme doctoral, l'équilibre à trouver entre des équipes à dominante technique et des équipes à dominante sociale et la nécessité d'opérer un important recrutement, le CNRS ne comptant aucun architecte dans ses titulaires sont 
autant de difficultés rencontrées à l'époque. Si certains architectes étaient ouverts à cet univers scientifique, les auditions révélaient également des réticences à faire correspondre création et recherche scientifique. Le dossier aboutit cependant et une section interdisciplinaire « Architecture, Urbanisme, Société » est créée. Elle commence à fonctionner en 1985, sous la présidence de Pierre Merlin et a été à l'origine d'équipes et de laboratoires comme l'IPRAUS. L'engagement des architectes reflète la diversité et la richesse d'un milieu professionnel créateur. Des équipes de sciences sociales sont intégrées, en particulier l'ISU, désormais Centre de Recherche sur l'Habitat installé à l'Ecole d'Architecture de Nanterre. Antoine Haumont y travaille et poursuit des recherches sur l'espace et les modes de vie, en particulier sur la mobilité, tout en restant jusqu'en 1989, chargé de mission au CNRS dans le Programme Interdisciplinaire de Recherche sur l'Environnement (PIREN).

\section{Le géographe, un rôle particulier dans le dialogue interdisciplinaire?}

12 Cette dernière expérience confirme la place particulière qu'Antoine Haumont a joué tout le long de son parcours professionnel en position d'interface entre acteurs, porteurs de savoirs différenciés. Sollicité pour introduire un savoir-penser l'espace dans l'équipe de l'ISU, il semble avoir été également mis à contribution pour penser les relations entre sciences sociales, sciences de l'ingénieur et architectes à travers différentes missions. Ce travail de mise en relation répondrait-il à l'idée que le géographe, traditionnellement vu comme opérateurs de synthèses et de transversalité sur le fond de ses analyses, porterait donc intrinsèquement une capacité à opérer d'autres formes de synthèses notamment organisationnelles et intercognitives ? Outre la mission de travailler avec des disciplines différentes, il a fallu à notre géographe composer avec les exigences des acteurs institutionnels et des opérateurs de l'aménagement, ce qui l'a placé en position d'interface entre le savoir académique et le savoir des décideurs. Pour Antoine Haumont, ses études de géographie et son expérience professionnelle à l'Université de Paris 7 lui ont donné les compétences pour dialoguer avec des savoirs épars. Le double cursus de l'époque, marqué par la géographie physique et la géographie humaine, permettait selon lui de se familiariser avec des notions et des concepts procédant d'un large éventail et de pouvoir dialoguer avec des spécialistes de la société comme avec ceux de la structure physique des milieux. A Paris 7, les relations perméables entre ce qui a été à un moment des sousdisciplines de la géographie ainsi que les relations de la géographie avec d'autres disciplines (mathématiques, sciences de la vie et de la terre) ont favorisé cette capacité de dialogue. Il rappelle également le rôle des "méthodes et techniques numériques ", de l'analyse spatiale, qui permettaient à un François Durand-Dastès de travailler aussi bien sur la climatologie que sur la société indienne.

13 Est-ce lié à une aura particulière et à des carrières professionnelles ou à des qualités de géographes, mais ce témoignage montre que des géographes étaient sollicités pour prendre en charge des responsabilités-clés. Pierre George avait été nommé directeur du groupe de travail sur l'habitat du Centre de Recherche Urbaine du Ministère de l'Equipement et Antoine Frémont directeur adjoint du département des sciences humaines et sociales du CNRS. Ces deux géographes ont eu un rôle dans le dialogue entre savoirs, le premier faisant appel à Henri Lefebvre pour dénouer la polémique 
entre habitat pavillonnaire et habitat collectif, le second mettant en musique une orientation interdisciplinaire de l'institution scientifique.

\section{L'aménagement urbain : une proximité entre l'action publique et la recherche scientifique}

Le parcours d'Antoine Haumont révèle en effet une proximité entre les géographes et l'action publique dans une période où l'État intervient fortement dans l'aménagement. Le Plan prend une nouvelle orientation dans les années soixante: au lieu d'être uniquement sectoriel comme il l'était dans l'immédiat après-guerre, il intègre la dimension spatiale et urbaine. C'est l'époque des villes nouvelles et des réseaux d'autoroute notamment. Pour développer sa capacité d'action, le Ministère de l'Équipement possède ses centres de recherches comme le Centre Scientifique et Technique du Bâtiment (CSTB) créé en 1947 et le Centre de Recherche sur l'Urbanisme (CRU) qui fait appel à des experts directement recrutés ou à travers des appels auxquels répondent les universitaires. On a vu par exemple, que le CRU avait commandé une étude sur les grands ensembles à l'Institut National d'Etudes Démographiques (INED) et une sur l'habitat pavillonnaire à l'ISU. Ces études, réalisées en interdisciplinarité, ont montré la diversité et la complexité de l'habitat, d'une manière inattendue pour les donneurs d'ordre. L'habitat collectif des grands ensembles était en cause. D'une part, cet habitat collectif apparaissait très positif aux décideurs de l'époque, conforme aux doctrines modernistes élaborées par Le Corbusier et la Charte d'Athènes, permettant une construction industrialisée massive et rapide et, enfin, apportant une surface habitable, le confort et l'accès aux réseaux (eau électricité, assainissement) aux populations de l'après-guerre. Cependant, d'autre part, les enquêtes menées dans la population montraient un attachement fort à l'habitat individuel et le souhait d'accéder au pavillonnaire, l'habitat dans les grands ensembles n'étant pensé que comme transitoire. Il y avait donc des dimensions sociales, culturelles voire psychologiques qu'il fallait intégrer à la politique du logement et de l'équipement. Bien que s'inscrivant dans une expertise et une commande publique fortement structurée, les recherches ouvraient la voie à un habitat plus diversifié. Antoine Haumont rappelle d'ailleurs que ces conclusions n'étaient pas facilement admises : l'idée que les individus s'habitueraient aux logements collectifs et qu'il fallait "leur apprendre à habiter " prédominait à l'époque. La commande publique de recherche a néanmoins permis de faire avancer la réflexion aussi bien du côté des autorités que du côté des scientifiques. Elle montre également une relation particulière au secteur public qui semble avoir changé depuis.

L'admission d'Antoine Haumont comme enseignant-chercheur à l'ENPC relève de cette logique puisque nous avons ici à faire à une institution nationale de formation dont l'objectif premier est de fournir à la puissance publique un corps d'ingénieurs pour l'équipement et l'aménagement des territoires notamment urbains. Elle révèle la nécessaire pluridisciplinarité dès lors qu'il est question d'aménager et les dimensions tant sociales que spatiales de l'action des ingénieurs.

Les choix éditoriaux des publications d'Antoine Haumont révèlent également le dialogue aussi bien avec les institutions publiques (Cahiers de l'IAURP, Annales de la recherche Urbaine, CRU, Documentation française, ...) qu'avec d'autres disciplines (Revue française de sociologie, Architecture d'Aujourd'hui, ...). 


\section{Une surdétermination politique} années cinquante et au début des années soixante, la guerre d'Algérie favorise les rapprochements dans un groupe de géographe engagés à gauche en même temps qu'elle fait découvrir à certains d'entre eux le Tiers-monde. Les questions du développement émergent à la suite de la décolonisation. Des affinités et des relations d'amitiés se nouent qui facilitent les mises en relation dans le monde académique et scientifique.

Le mouvement de mai 68 a également des conséquences pour cette génération de géographes. L'une est institutionnelle au niveau de l'enseignement supérieur avec l'éclatement de la Sorbonne en plusieurs nouvelles universités. Un groupe de géographes se constitue alors pour rejoindre l'Université de Paris 7 parmi lesquels Jean Dresch, Fernand Joly, Olivier Dollfus, François Durand-Dastès, Antoine Haumont. Ces géographes participèrent à l'organisation d'une unité d'enseignement de recherche innovante qui associait directement les géographes, les historiens et les économistes. Un enseignement professionnel sur l'aménagement avait été mis en place.

Mai 68 a également pour effet de favoriser les sciences sociales et par ricochet, la géographie: les réponses de l'aménagement, très techniques et fonctionnelles, montraient déjà leurs limites tout en restant dominantes. La relation de l'espace privé de l'habitant à l'espace public de sociabilité n'est pas prise en compte; plus globalement, c'est le mode de l'habiter qui est négligé par les ingénieurs. La mobilité et l'habitat n'apparaissent plus seulement comme des problèmes techniques mais aussi sociaux comme le montre Gabriel Dupuy, centralien et ingénieur de profession jusqu'en 1973 avant de passer à l'Université et de réaliser une thèse en 1977. La ville fonctionnelle, l'urbanisme de dalle, commencent à être dénoncés. C'est dans ce contexte que des Écoles d'ingénieur comme celle des Ponts et Chaussées affichaient la nécessité d'ouvrir leur enseignement aux sciences sociales et notamment à la géographie. Cette évolution entamée dès le milieu des années soixante est renforcée après 1968 et reprise avec l'alternance politique de 1981. En effet, la recherche d'une alternative à l'action publique conduit à des modifications institutionnelles, favorisent des recompositions disciplinaires et ont pu bénéficier alors aux sciences sociales.

On notera dans ce récit que certains lieux de rencontre et d'échange ont favorisé la mise en relation des personnes. L'Institut de Géographie a ainsi pu permettre la constitution d'un capital relationnel et favoriser la curiosité pour sortir des cadres institutionnels de sa discipline. L'envie d'être géographe citoyen en partant de sa discipline pour répondre à des besoins sociaux portés par l'action publique explique également le parcours d'Antoine Haumont, représentatif en cela de nombre de géographes qui n'ont pas douté de ce qu'ils pouvaient apporter aux autres disciplines et aux décideurs, ni sur leur rôle de passeur entre savoirs. 


\section{BIBLIOGRAPHIE}

Godelier M, 1982. Les sciences de l'homme et de la société en France, analyse et propositions pour une politique nouvelle. Rapport au ministre de la Recherche et de l'Industrie, La Documentation française, Paris, $611 \mathrm{p}$.

Haumont A., 1967. Les quartiers dans trois communes de la banlieue parisienne. Cahiers de l'IAURP, vol 7, $25 \mathrm{p}$.

Haumont A., 1968. Les agglomérations françaises de plus de 50000 habitants. Revue française de Sociologie, $28 \mathrm{p}$.

Haumont A., 1969. Les équipements sportifs dans la Région Parisienne. Paris, ISU/Districts de la Région Parisienne, 205 p.

Haumont A., 1976. Les espaces du travail aujourd'hui. Architecture d'Aujourd'hui, nº 188.

Haumont A., 1989. Les habitants des lotissements : des identités variées. Villes en parallèle, La ville fragmentée, $n^{\circ} 14$, p. 172-181.

Haumont A., 1991. L'Ile de France, développement, aménagement, environnement. Les Annales de la Recherche Urbaine, $\mathrm{n}^{\circ} 50$.

Haumont A., 1996. L'Equipement culturel des villes. Annales de la Recherche Urbaine, 70.

Institut de Sociologie Urbaine, 1968. Propositions de recherches sur la vie urbaine. Revue de Sociologie Urbaine, vol IX, n² 2, avril-juin 1968, p. 151-166.

Lefebvre H., 1962. Critique de la vie quotidienne, fondements d'une sociologie de la quotidienneté. L'Arche.

Raymond H., Haumont N., Dezès M.G., Haumont A., 1966. L'Habitat Pavillonnaire. L'Harmattan, préface de Henri Lefebvre, coll. Habitat et Société, Paris.

\section{AUTEUR}

\section{ALEXIS SIERRA}

Alexis Sierra, alexisierra2001@yahoo.fr, est maître de conférences en géographie à l'université Cergy-Pontoise-IUFM. 